\title{
Early and late arrhythmias after the Fontan operation: predisposing factors and clinical
} consequences

\author{
Marc Gewillig, Richard K Wyse, Marc R de Leval, John E Deanfield
}

\begin{abstract}
Objective-To study the incidence, predisposing factors, and clinical significance of arrhythmias early and late after the Fontan operation for congenital heart disease.
\end{abstract}

Patients and methods-All 104 consecutive patients undergoing Fontan repair from 1975 to 1988 were studied retrospectively. Hospital records were reviewed for perioperative arrhythmia. Clinical information and annual electrocardiograms were available for all 78 hospital survivors during a follow up of up to 13 years (mean 3.7 years). Ambulatory electrocardiographic monitoring was performed in 67 patients (81\%).

Results-Eleven patients (10.6\%) developed a perioperative tachycardia (eight, atrial flutter; three, His bundle tachycardia). Multivariate analysis showed that raised preoperative mean pulmonary artery pressure and low aortic saturation were significant risk factors for the development of atrial flutter $\left(r^{2}=0.32, p=0.0001\right)$ but not for His bundle tachycardia. Despite intensive medical treatment 10 of these 11 patients died. At the last visit $72(92 \%)$ of the 78 patients were in sinus rhythm on their standard 12 lead electrocardiogram. Junctional rhythm was present in three patients, two patients had atrial flutter, and one had a paced rhythm. Ambulatory monitoring did not show important bradycardia or ventricular arrhythmias. Actuarial survival free of supraventricular arrhythmia was $82 \%$ at eight years after operation. Multivariate analysis identified older age, increased right atrial size, and raised mean preoperative pulmonary artery pressure as risk factors for arrhythmia during intermediate follow-up $\left(r^{2}=0.46\right.$, p $<0.001$ ). Late tachycardias, in contrast to those occurring in the perioperative period, were not associated with an increased mortality.

Conclusions-Except for His bundle tachycardia in the perioperative period, early and late arrhythmias after a Fontan operation seem to be a consequence of adverse preoperative and postoperative haemodynamic function. The perioperative outcome is therefore poor even when the patient can be restored to sinus rhythm. Medical and surgical modifications to improve the haemodynamic disturbances associated with arrhythmias are therefore indicated.

In 1971 Fontan described an operation to connect the right atrium to the pulmonary artery in patients with tricuspid atresia, without incorporating an adequate right ventricle in the circulation. ${ }^{1}$ Modifications of this procedure are being used as the definitive surgical repair in patients with an increasingly wide range of complex congenital heart disease. All the various surgical techniques that have been advocated involve atrial surgery and result in a chronic increase in the systemic venous atrial pressure. $^{23}$ Both factors predispose to supraventricular arrhythmia. ${ }^{4-7}$ The acute and chronic haemodynamic consequences of such arrhythmia, producing a rise in the pulmonary venous atrial pressure in a circulation that usually lacks a subpulmonary ventricle, may be serious. Little information on arrhythmia after the Fontan operation and its consequences has been published. $^{8}$ We therefore investigated a consecutive series of patients undergoing Fontan repair, to study the incidence, time course, and predisposing factors to early and late arrhythmia, and to identify the circumstances in which this may contribute to increased morbidity and mortality.

\section{Patients and methods}

PATIENTS

All 104 patients (62 male, 42 female) undergoing a Fontan operation between April 1975 and March 1988, and who were resident in the United Kingdom, were included in the study. Initially we used the strict selection criteria of Choussat et al $^{9}$ but over the course of the study several modifications and exceptions were introduced. As a result, a wide variety of anomalies and surgical connections are represented in the patient population (table 1). Early on, valved conduits from the right atrium to right venticle or right atrium to pulmonary artery were inserted but later only direct atriopulmonary or total cavopulmonary connections were performed. Biographical information, morphological diagnosis, details of previous surgery, haemodynamic variables before Fontan operation, surgical technique, and follow-up status were available in all patients. 
Table 1 Morphological and surgical characteristics

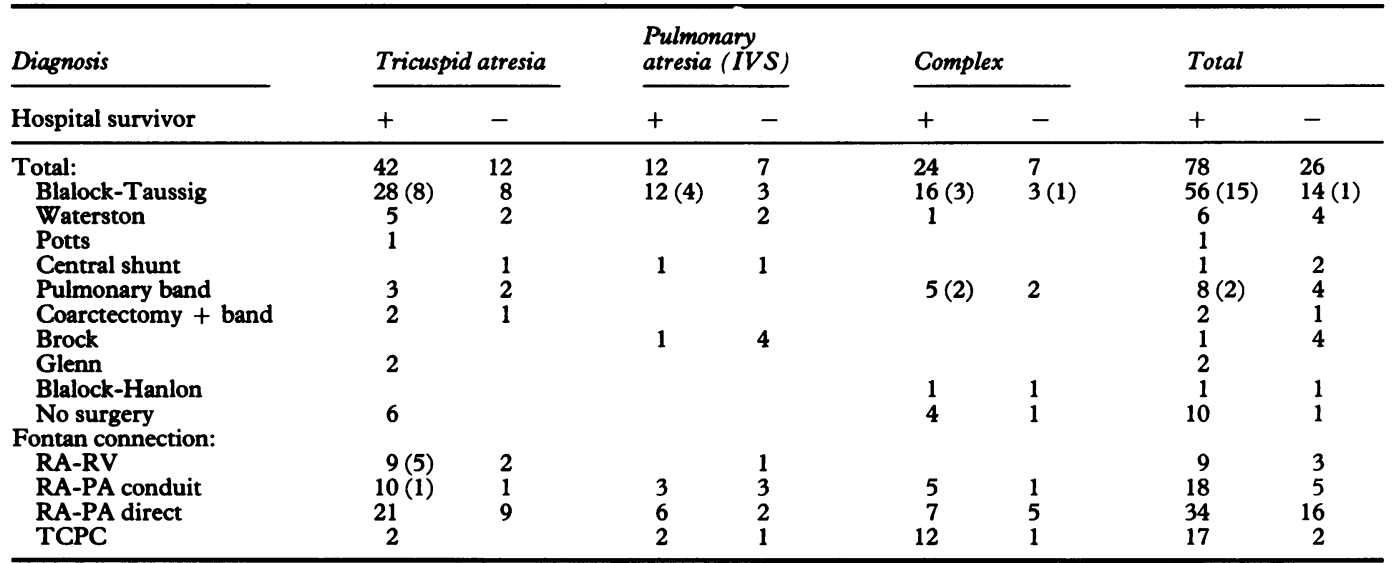

IVS, intact ventricular septum; RA, right atrium; RV, right ventricle; TCPC, total cavopulmonary connection. Second similar operations are indicated in parentheses.

\section{METHODS}

Hospital records, including drug charts and notes from the intensive care unit, were reviewed for arrhythmia which occurred before and during admission to hospital for operation and required intervention such as pacing, cardioversion, or antiarrhythmic treatment. Twelve hours after operation and just before the onset of an arrhythmia we assessed the patient's haemodynamic condition by examining right atrial pressure, transpulmonary gradient, diuresis, blood pressure, inotropic support, and the requirement for dialysis or abdominal counterpulsation.

Follow up information was obtained in all patients, either by outpatient assessment or by contact with the patient's family and primary physician. Patients and parents were questioned about the functional status and symptoms of arrhythmias. Follow up records and serial electrocardiograms obtained at least annually were also reviewed for symptoms and objective evidence of rhythm disturbance.

\section{AMBULATORY MONITORING}

In 67 patients 24 hour ambulatory electrocardiographic monitoring was performed $0 \cdot 2-13$ (mean 3.4) years after the Fontan procedure. Care was taken to monitor all patients in whom clinical follow up suggested an arrhythmia. Oxford Medilog 2 channel recorders were used (CM1 and CM5 leads) and tapes were analysed visually at 60 times real time with an Oxford Medilog playback unit. The basic cardiac rhythm and the presence of arrhythmias or conduction defects were noted. Patients were considered to be in stable sinus rhythm if this was present for $>90 \%$ of the 24 hour period. Records were classified as intermittent sinus and junctional rhythm if junctional escape rhythm was present in $10-50 \%$ of the recording and junctional rhythm if this was present $>50 \%$ of the time. Atrial and ventricular extrasystoles were counted and ranked as proposed by Nagashima et $a l^{10}:<4,5-9,40$ 49 , or $>50$ extrasystoles per 24 hour period. The presence of complex forms including multiform beats, couplets, and runs of ventricular tachycardia $(\geqslant 3$ consecutive ventricular extrasystoles at a heart rate of $>120$ beats $/ \mathrm{min}$ ) was also noted.

\section{EXERCISE TESTING}

Supine graduated exercise testing on a bicycle ergometer was performed by $73 \%$ (46 patients) of the survivors over the age of 5.5 years in whom stress testing was considered feasible. Patients were exercised for five minutes at progressively increasing workloads from $0.25 \mathrm{~W} / \mathrm{kg}$ to $1.5 \mathrm{~W} / \mathrm{kg}$ with $0.25 \mathrm{~W} / \mathrm{kg}$ increments per minute. Heart rate and rhythm were monitored continuously. Rate was expressed as a function of an age-predicted value derived from data in 28 age-matched controls studied in our laboratory.

\section{HAEMODYNAMIC STATUS}

Routine postoperative cardiac catheterisation was not performed but catheterisation data were available in $28(32 \%)$ of survivors. In 47 patients $(60 \%)$ echocardiography was used to study ventricular size and contractility and atrial size. Except for the patients with a total cavopulmonary connection, the length and width of the atria were measured from the apical view and their product divided by the body surface area to obtain an index of atrial size. In the patients who developed chronic atrial arrhythmias data were obtained from echocardiograms recorded when the patient was last seen in sinus rhythm.

\section{STATISTICAL ANALYSIS}

Data were stored in a SIR (version 2) database on an Amdahl mainframe computer and analysed with SAS statistical packages (SAS Institute, Cary, North Carolina, USA). All data were expressed as mean with standard deviation and/or range. Actuarial analysis of late survival free of supraventricular arrhythmias (as detected by any method and using the time of operation as time zero), was made by the Kaplan-Meier method. Univariate analysis and multiple regression techniques were used to determine risk factors for perioperative and late postoperative arrhythmias. Chi squared statistics were used to study differences of proportions; independent testing and re- 
peated-measures analysis of variance were used to compare the exercise results of the Fontan patients with those of age-matched controls.

\section{Results}

\section{PATIENT CHARACTERISTICS}

Table 1 summarises the morphological and surgical characteristics of the patients. Almost all (93 patients $(89 \%)$ ) had undergone palliation before the Fontan procedure, either by banding of the pulmonary trunk, by shunt procedures, or by other miscellaneous interventions. Their mean (SD) age at Fontan operation ranged from 0.9 to 18.3 years $(7 \cdot 12$ (3.9)) and the $78(75 \%)$ hospital survivors were followed for $0 \cdot 2-13$ years $(3 \cdot 7(3 \cdot 3))$. At the last assessment, 27 patients $(36 \%)$ were taking cardiac medication (digitalis in 18, diuretics in seven, vasodilators in nine, other antiarrhythmic agents in nine, anticoagulants in five). Sixty four patients were in New York Heart Association class I, 10 in class II, and one in class III. Three patients had died during follow up-two at re-operation and one two months after Fontan operation.

\section{PERIOPERATIVE ARRHYTHMIA: BRADYCARDIA}

During the first 24 hours after operation (mean onset 6.3 hours) eight $(7 \cdot 7 \%)$ patients had a bradycardia requiring specific treatment. In three it was sinus bradycardia (rates 50, 62, 68 beats/min) and in five patients a slow junctional escape rhythm (range 50-72 beats/min, mean 58). Atrial pacing (five patients) and/or isoprenaline (seven patients) was effective and improved the haemodynamic condition of all the patients. In six treatment was stopped after 872 hours (mean 31) but two patients with persistent sinus bradycardia were discharged on oral isoprenaline for two and three months respectively ( 60 and 67 beats/min under treatment). Two of the patients with early significant bradycardias subsequently developed atrial flutter 25 and 72 hours later with accompanying rapid deterioration of their haemodynamic condition. One died and the other was restored to sinus rhythm after amiodarone and electrical cardioversion. He has remained in intermittent sinus and junctional rhythm over a four year follow up period on amiodarone. Atrial flutter recurred after some months on the one occasion when medication was stopped. Of the remaining six patients, one has developed atrial flutter 14 months after Fontan. On ambulatory monitoring (1-7, mean 3.5 years after surgery) three of these patients were in sinus rhythm with frequent ( $>200$ in 24 hours) atrial extrasystoles. This feature was seen in only two of 55 patients with no perioperative bradycardia in whom ambulatory electrocardiographic monitoring was performed. Thus perioperative bradycardia though not associated with increased mortality was associated with an increased incidence of late arrhythmias $(p<0.001)$.

\section{ATRIOVENTRICULAR BLOCK}

Persistent complete atrioventricular block after the Fontan operation in two patients with a double inlet ventricle. Both were treated in the perioperative period with atrioventricular sequential pacing and a DDD pacemaker was implanted in one patient after one month because of a slow junctional rhythm ( 30 beats/ min). The other patient had considerable left ventricular dysfunction and poor haemodynamic function with a spontaneous ventricular rate of 60 beats $/ \mathrm{min}$. The clinical condition did not improve with atrioventricular sequential pacing and he died two months after operation while awaiting cardiac transplantation. No other patient developed atrioventricular block.

\section{TACHYCARDIA}

Tachycardias developed in 11 patients during the early postoperative period. One girl developed atrial fibrillation as she was weaned from bypass (right atrial pressure $17 \mathrm{~mm} \mathrm{Hg}$ ). Despite an acceptable ventricular rate on multiple antiarrhythmic agents and several attempts at cardioversion she remained in a low output state. While she was being prepared for a take-down of her Fontan operation four hours after the initial operation she died in ventricular fibrillation. A further five patients developed atrial flutter in the first 24 hours after operation (1-24, mean 9 hours). In all the arrhythmia was preceded by poor haemodynamic function reflected by high right atrial pressure (19-23, mean $21 \mathrm{~mm} \mathrm{Hg}$ ), low urinary output and the need for maximal inotropic support, and abdominal counterpulsation (two patients). All died 1-8 days after the Fontan operation despite early conversion to sinus rhythm in three patients (overdrive pacing 1 , electrical cardioversion 2) and improvement in ventricular rate in two with amiodarone. A further patient collapsed suddenly while in sinus rhythm after successful weaning from the ventilator half an hour after extubation on the third postoperative day. After resuscitation he was found to be in atrial flutter and was successfully converted by overdrive pacing to sinus rhythm. Despite the absence of any further arrhythmia he died 20 days later of a pulmonary infection. Only one patient, who developed atrial flutter three days after surgery, survived. He was successfully returned to sinus rhythm by electrical cardioversion after treatment with intravenous amiodarone.

A further three patients developed a $\mathrm{His}$ bundle tachycardia (rate 162-200, mean 175 beats/min) within three hours of operation. Before the tachycardia all three patients had satisfactory haemodynamic function as reflected by a low ( $<15 \mathrm{~mm} \mathrm{Hg}$ ) systemic venous pressure, good cardiac output, and diuresis with minimal support. The tachycardia rate was not successfully controlled (by digoxin and atrioventricular sequential pacing) in one patient and despite good early haemodynamic function he died three hours after onset of the arrhythmia. In the second and third patients amiodarone and overdrive atrioventricular sequential pacing slowed the automatic tachycardia and improved the haemodynamic condition, but despite this both patients died one and seven days later at attempted takedown of the Fontan operation. 
Multivariate analysis produced a significant model $\left(r^{2}=0.32, p<0.0001\right)$ predicting the occurrence of perioperative atrial flutter with a mean preoperative pulmonary artery pressure of $>16 \mathrm{~mm} \mathrm{Hg}(p=0.002)$, low preoperative aortic saturation ( $p=0.041$ ), and young age at Fontan operation $(p=0.086)$. There were too few patients with $\mathrm{His}$ bundle tachycardia to perform a risk factor analysis. However, there were no specific features that identified these patients even on retrospective analysis, and these patients clearly did not fit the profile of those patients who developed perioperative atrial flutter.

\section{RESULTS DURING INTERMEDIATE FOLLOW UP} Three of 78 hospital survivors died during follow up. None of the deaths, however, seemed to be related to an arrhythmia. One patient with double inlet left ventricle had borderline preoperative haemodynamic function (mean pulmonary artery pressure 27 $\mathrm{mm} \mathrm{Hg}$, left ventricular dysfunction with atrioventricular valve regurgitation) and the result of his Fontan operation was poor (mean right atrial pressure $20 \mathrm{~mm} \mathrm{Hg}$ one week after operation). He had developed complete atrioventricular block postoperatively (see above) but the haemodynamic condition had not improved with atrioventricular sequential pacing. He died two months after the Fontan operation. Two other patients died $5 \cdot 1$ and $7 \cdot 5$ years after the Fontan repair, both at the time of the second replacement of a right atrial to pulmonary artery valved conduit. Both patients had 24 hour ambulatory monitoring performed after the first operation and before the second operation. One was in sinus rhythm throughout and the other had short periods of supraventricular tachycardia alternating with sinus bradycardia and pauses of up to two seconds. He had a pacemaker inserted because of dizzy spells. However, this failed to improve his symptoms, which were then attributed to low cardiac output secondary to severe obstruction of his conduit. No other cases of late bradycardia were detected.

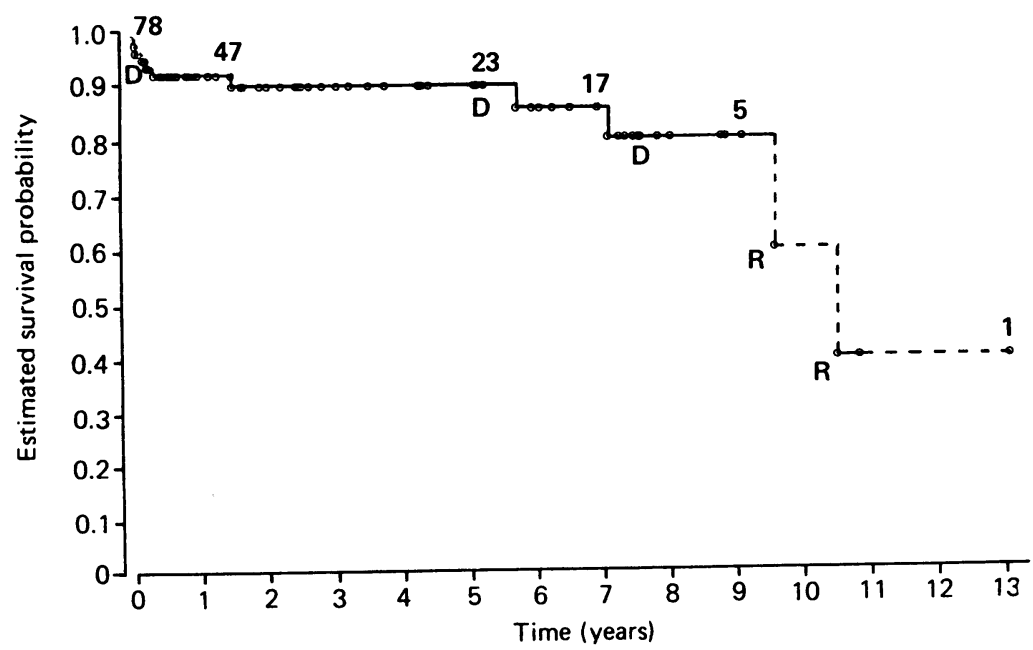

Figure 1 Actuarial survival free of supraventricular tachycardia after the Fontan operation (Kaplan-Meier method with time of operation as time zero). Each patient withdrawn is represented by a circle; the three late deaths $(D)$ are indicated. $R=$ redo Fontan with atrial flutter in early convalescence $(n=2)$.
Eleven patients $(14 \%$ of postoperative survivors) experienced a paroxysmal tachycardia during their late postoperative course as detected by clinical examination, routine electrocardiography, ambulatory monitoring, or exercise testing. One of these was the only survivor of a tachycardia in the perioperative period (see above). Atrial flutter recurred a few months after an attempt at stopping amiodarone treatment. It was restarted and he has been successfully maintained in intermittent sinus and junctional rhythm. A further five patients developed clinical or electrocardiographic evidence of tachycardia shortly after discharge from hospital, all while doses of digitalis and diuretics were being tapered off (mean interval after operation 1.9 months, range 2 weeks to 4 months). In three there was only clinical evidence of short bouts of tachycardia and symptomatic palpitation with no haemodynamic upset. Repeated ambulatory electrocardiographic monitoring did not detect objective evidence of an arrhythmia in these patients. The two other patients were admitted to hospital in atrial flutter with 2:1 atrioventricular block and a ventricular rate of 180 beats $/ \mathrm{min}$. Both were cardioverted to sinus rhythm. There has been no subsequent arrhythmia on chronic medication with digitalis in one or flecainide in the other. In three patients late tachycardias were related to problems with conduit obstruction. One patient with severe obstruction of his second valved conduit between the right atrium and right ventricle had short runs of supraventricular tachycardia (rate 120 beats/min) during ambulatory electrocardiographic monitoring. He died at second reoperation. Two other patients developed atrial flutter with only mild haemodynamic upset one and three months after reoperation for conduit obstruction. Sinus rhythm was re-established and maintained with amiodarone in one patient and the ventricular rate was controlled with flecainide in the other. Two patients developed new atrial flutter more than a year after the Fontan operation. One of these patients had been treated for sinus bradycardia in the perioperative period and had been discharged on oral isoprenaline for three months. Postoperative treatment with diuretics was stopped only at a 14 months evaluation and when she was reviewed at 22 months she was noted to be in atrial flutter with a ventricular rate of 140 beats $/ \mathrm{min}$. This change in rhythm was associated with a significant increase in ventricular size and impaired left ventricular contractility. The other patient was admitted to an emergency department seven years after his Fontan operation: he had atrial flutter and a ventricular rate of 220 beats/min associated with impaired haemodynamic function. He had noticed occasional episodes of palpitation over the previous month with one syncopal episode. He was treated with intravenous verapamil, which initially produced some slowing of the heart rate which then, however, deteriorated to asystole. He was resuscitated but experienced a severe multiorgan ischaemic insult and has made only a slow recovery. 


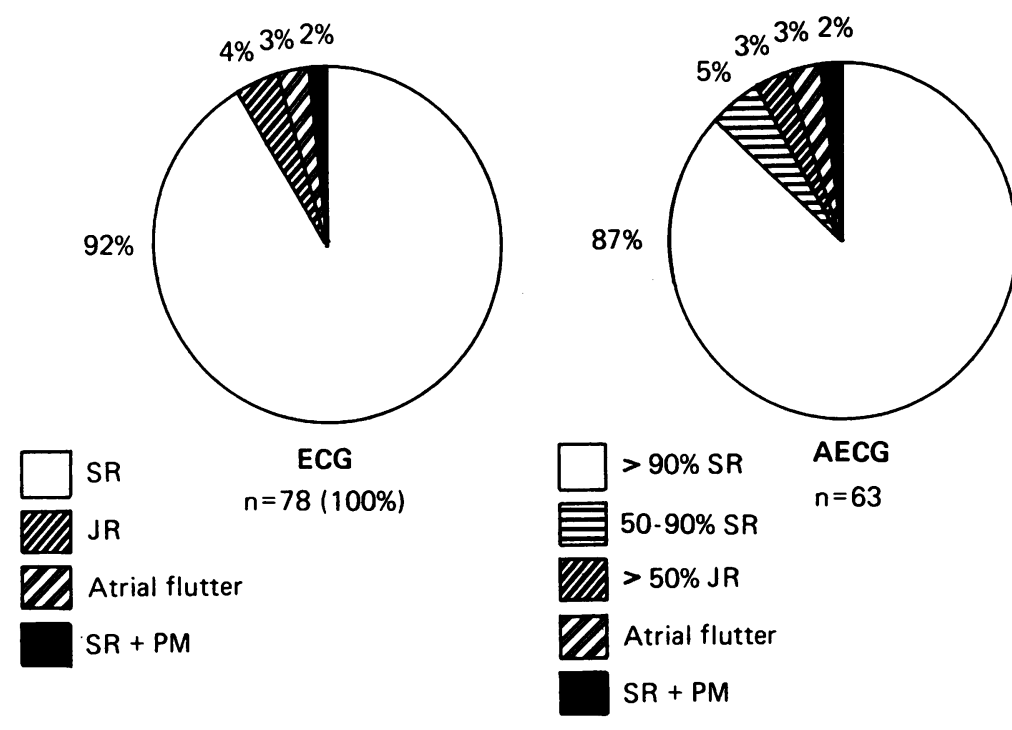

Figure 2 Cardiac rhythm in Fontan patients on last electrocardiogram (ECG) and on 24 hour ambulatory electrocardiographic monitoring ( $A E C G) . J R$, junctional rhythm; SR, sinus rhythm; PM, pacemaker.

Actuarial analysis showed that survival free of tachycardias detected by any method was $89 \%$ at five years and survival at eight years after a successful Fontan operation was $82 \%$ (fig 1). Nine of the 11 patients with late tachycardia reported symptoms (five mild, three moderate, one severe).

ROUTINE ELECTROCARDIOGRAMS

At last visit, 72 of the $78(92 \%)$ patients were in sinus rhythm on their standard 12 lead electrocardiograms (fig 2). Junctional or low right atrial rhythm was present in three patients, two of whom had left isomerism. Two patients had atrial flutter and one had a paced rhythm. First degree atrioventricular block was present in three patients, all of whom were taking digitalis.

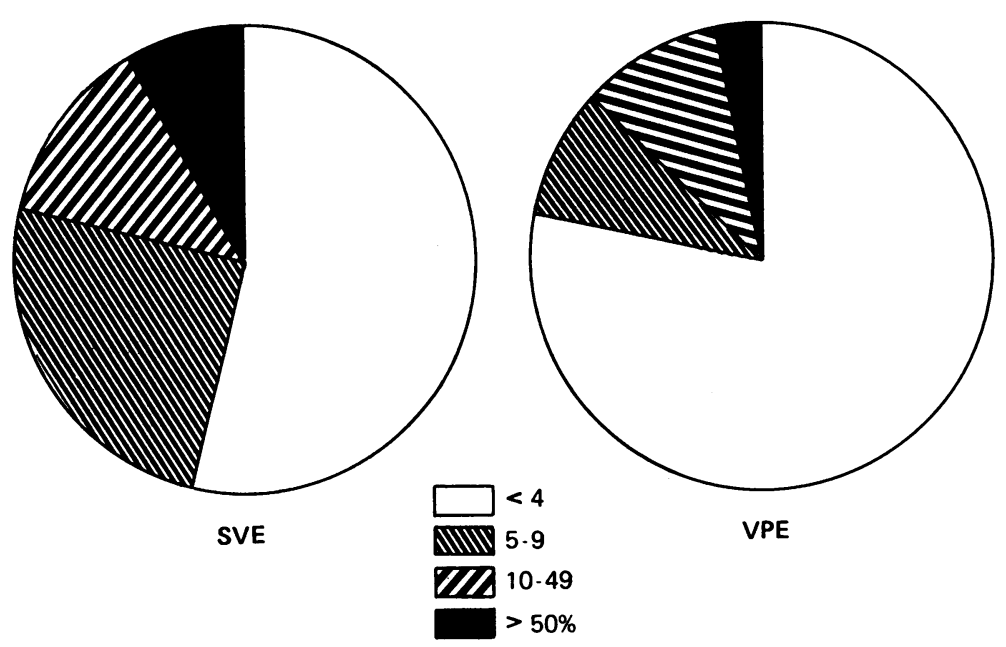

Figure 3 Supraventricular and ventricular extrasystoles (SVE and VE) on a 24 hour ambulatory electrocardiographic monitoring. Numbers refer to number of patients in each subgroup.
24 HOUR AMBULATORY ELECTROCARDIOGRAPHIC MONITORING

Ambulatory monitoring was performed in 67 hospital survivors $3.5(3.3)$ years after Fontan operation and $63(81 \%$ of the total group) recordings were of adequate quality. Fifty five $(87 \%)$ of these patients were in sinus rhythm. The remainder were in sinus rhythm with intermittent junctional escape (three patients), established junctional rhythm (2 patients), chronic atrial flutter (two patients), and one paced rhythm. Eight patients had a wandering atrial pacemaker during recordings. Figure 3 summarises the atrial and ventricular extrasystoles detected by ambulatory monitoring. Only two patients had more than 50 ventricular extrasystoles. All were isolated and uniform. One patient showed an episode of ventricular bigeminy at rest and one patient had two ventricular couplets. Five patients $(8 \%)$ had more than 50 supraventricular extrasystoles in the $\mathbf{2 4}$ hour recording. No significant bradycardias or atrioventricular block were seen. Ambulatory monitoring detected evidence of arrhythmia with bouts of supraventricular tachycardia in only one patient and multiple recordings failed to detect arrhythmias in three patients with symptoms of palpitation and clinically documented tachycardias.

\section{EXERCISE TESTING}

All patients except one were in sinus rhythm throughout the exercise test. One patient with chronic atrial flutter had frequent uniform ventricular extrasystoles at rest which disappeared as soon as she started to exercise. Similarly, two other patients had frequent atrial extrasystoles at rest but these disappeared on exercise. No new ventricular arrhythmia was documented. At the highest workload of $1.5 \mathrm{~W} / \mathrm{kg}$ the controls had a heart rate of 124 (18) beats per minute. The heart rate at rest was significantly higher in the Fontan patients than in the age-matched controls $(p<0.01)$. This difference disappeared as soon as exercise started so that at all levels of exercise the heart rate in the Fontan patients was similar to that seen in age-matched controls.

FACTORS ASSOCIATED WITH LATE ARRHYTHMIA

Table 2 summarises factors that were assessed to determine risk factors for late arrhythmia. Multivariate analysis produced a significant model $\left(\mathrm{r}^{2}=0.46, \quad \mathrm{p}<0.001\right)$ predicting occurrence of a tachycardia during intermediate follow up with older age $(p<0.01)$, larger index of atrial size $(p<0.05)$, and a preoperative mean pulmonary artery pressure $>20 \mathrm{~mm} \mathrm{Hg} \quad(\mathrm{p}<0.01)$. Occurrence of frequent ( $>50$ per minute) atrial extrasystoles was predicted by a model $\left(\mathrm{r}^{2}=0.33\right.$, $\mathrm{p}<0.005)$ which used time since Fontan repair $(\mathrm{p}<0.003)$ and preoperative mean pulmonary artery pressure as variables $(p<0.02)$. In this study, we were unable to show a relation between the occurrence of arrhythmias during intermediate follow up and either the underlying cardiac lesion or the surgical technique. 
Table 2 Factors included in the multivariate analysis for late tachycardia

\begin{tabular}{|c|c|c|c|}
\hline \multirow[b]{2}{*}{ No } & \multicolumn{2}{|c|}{ Late tachycardia } & \multirow[b]{2}{*}{$t$ test $(p)$} \\
\hline & Absent & Present & \\
\hline $\begin{array}{l}\text { Age at Fontan operation }(y) \\
\text { Follow up }{ }^{\star}(y) \\
\text { Age }{ }^{\star}(y) \\
\text { Preoperative haemodynamics }(n=78) \text { : }\end{array}$ & $\begin{array}{r}7.3(3.8) \\
3.5(3.1) \\
10.9(5.3)\end{array}$ & $\begin{array}{r}10 \cdot 1(4 \cdot 1) \\
4 \cdot 3(4 \cdot 2) \\
14 \cdot 4(5 \cdot 5)\end{array}$ & $\begin{array}{c}<0.05 \\
\text { NS } \\
<0.05\end{array}$ \\
\hline $\begin{array}{l}\text { Mean PA pressure }(\mathrm{mm} \mathrm{Hg}) \\
\text { PA pressure }>20 \mathrm{~mm} \mathrm{Hg} \\
\text { Qp/Qs } \\
\text { LVEDP }(\mathrm{mm} \mathrm{Hg}) \\
\text { Aortic saturation }(\%)\end{array}$ & $\begin{array}{r}14 \cdot 0(4 \cdot 3) \\
5 / 67 \\
1 \cdot 6(1 \cdot 1) \\
9 \cdot 3(3 \cdot 3) \\
83 \cdot 8(5 \cdot 2)\end{array}$ & $\begin{array}{r}13.9(5 \cdot 6) \\
3 / 11 \\
1 \cdot 7(0 \cdot 6) \\
8 \cdot 1(4 \cdot 9) \\
86 \cdot 2(4 \cdot 1)\end{array}$ & $\begin{array}{l}\text { NS } \\
<0 \cdot 01 \\
\text { NS } \\
\text { NS } \\
\text { NS }\end{array}$ \\
\hline $\begin{array}{l}\text { Echo data: } \\
\text { LVEDD }(\%) \\
\text { Fractional shortening }(\%) \\
\text { RA width } \times \text { length } / \text { BSA } \\
\text { LA width } \times \text { length } / \text { BSA } \\
\text { Cardiothoracic index } \\
\text { Tallest } P \text { on ECG }(\mathrm{mm})\end{array}$ & $\begin{array}{l}115(15) \\
33 \cdot 3(5 \cdot 9) \\
26 \cdot 8(9 \cdot 9) \\
9 \cdot 6(2 \cdot 9) \\
0.52(0.05) \\
3.0(1 \cdot 1)\end{array}$ & $\begin{array}{l}122(13) \\
30 \cdot 0(6 \cdot 6) \\
36 \cdot 6(9 \cdot 0) \\
11 \cdot 2(4 \cdot 6) \\
0 \cdot 55(0 \cdot 13) \\
2 \cdot 5(1 \cdot 6)\end{array}$ & $\begin{array}{r}\text { NS }(n=44) \\
\text { NS }(n=44) \\
<0.05(n=47) \\
\text { NS }(n=47) \\
\text { NS }(n=78) \\
\text { NS }(n=78)\end{array}$ \\
\hline
\end{tabular}

BSA, body surface area; LA, left atrium; LVEDD, left ventricular end diastolic dimension as $\%$ of normal for $\mathrm{BSA}^{27}$; LVEDP, left ventricular end diastolic pressure; PA, pulmonar artery; RA, right atrium. *Time to or age at end of follow up or occurrence of tachycardia.

\section{Discussion}

The Fontan operation and its modifications involve atrial surgery and may result in a circulation that functions at a chronically raised right atrial pressure. Both these factors are known to increase the likelihood of supraventricular arrhythmias. Our results show that the circulation after the Fontan operation is vulnerable to such tachycardias, particularly in the early postoperative period when they are highly associated with poor outcome. Late tachycardias, however, were better tolerated and did not provoke sudden death. These results contrast with published data from the Mayo Clinic in which 6/23 (26\%) of the late deaths after Fontan surgery were sudden or attributed to arrhythmias. ${ }^{11}$

\section{PERIOPERATIVE ARRHYTHMIA}

The incidence of perioperative arrhythmias is undoubtedly underestimated because of the retrospective design of our study. Loss of sinus rhythm or ectopic rhythms that were transient and well tolerated will have been missed. Nevertheless, life threatening rhythm disturbances were noted in $10 \%$ of the patients. Without preoperative monitoring it is not possible to determine whether arrhythmias were already significantly more common in this group before the Fontan surgery. Certainly, some patients undergoing surgery have atrial isomerism, which is known to be associated with structural and electrophysiological abnormalities and loss of sinus rhythm. ${ }^{12}$

Two different patterns of perioperative tachycardias were noted. The most common was atrial flutter which was associated with borderline preoperative and postoperative haemodynamic function. Atrial flutter therefore seemed to be a marker that identified patients with adverse risk factors; moreover, when it did occur, atrial flutter triggered a rapid deterioration and accelerated an already existing decline in haemodynamic function. Acute and chronic changes in the electrophysiological properties of the atria in response to stretching and increase in pressure are well recognised. In the dog model, Boyden and Hoffman showed both increased susceptibility to initiation of atrial flutter and increased duration of arrhythmia in distended atria. ${ }^{6}$ Potential substrates for arrhythmia have also been noted in studies on diseased human atria. ${ }^{13}$ Furthermore, extensive atrial surgery as in the Mustard and Senning operation increases early arrhythmia. Puga et al and Matsuda et al reported a high incidence of severe atrial flutter after a modified Fontan technique in which a cavocaval channel was created with atrial wall for patients with left atrioventricular valve atresia or a single atrioventricular valve. ${ }^{14}$ Sinus rhythm optimises systemic ventricular function. Though atrioventricular synchrony may not be required for survival, ${ }^{16}$ the loss of the atrial contribution to systemic ventricular filling and increase in atrial pressure secondary to arrhythmias may seriously impair cardiac output after the Fontan operation. ${ }^{17}$

The poor outcome of the patients with postoperative atrial flutter emphasises the impact of patient selection on the results after Fontan operation. Both a raised preoperative mean pulmonary artery pressure and low aortic saturation were identified as significant risk factors for early atrial flutter. A raised pulmonary artery pressure is associated with some medial hypertrophy, which predisposes to increased pulmonary vascular resistance or reactivity or both. ${ }^{18}$ The effects of hypoperfusion on the developing pulmonary circulation may be as deleterious as those of hyperperfusion and pulmonary hypertension. The proximal elastic arteries develop less elastin than normal, while the intra-acinar arteries are usually smaller and have a thinner coat. Pulmonary arterial thromboses can occur and they account for eccentric areas of intimal fibrosis. Moreover the development of the alveoli is impaired when the lung is severely hypoperfused. ${ }^{19} 20$ Extrapolation of vascular resistance at a higher flow condition (that is, full systemic flow as after a Fontan operation) from a resistance calculated in a low flow condition (that is $\mathrm{Qp} / \mathrm{Qs}<0.7)$ requires some assumptions such as adequate compliance of the pulmonary vascular bed. ${ }^{21}$ This study and other observations have led our surgeons often to prefer a shunt procedure to a Fontan operation in cyanotic patients.

A second but less frequent tachycardia noted in the early postoperative period was $\mathrm{His}$ bundle tachycardia. In contrast to atrial flutter this was not predicted by preoperative or postoperative haemodynamic condition. Similar arrhythmias have been seen after other cardiac operations involving manipulation of the area around the atrioventricular node, including repair of a ventricular septal defect and tetralogy of Fallot. ${ }^{22}$ Such arrhythmias were particularly poorly tolerated in the Fontan circuit, in which only minor increases in left atrial pressure can be disastrous. Such an increase resulted in rapid deterioration and death in our patients. Presumably, His bundle tachycardia is related to some aspects of the surgery itself. The Fontan operation requires atrial manipulation and suturing, and damage to the atrioventricular node and its blood 
supply is one possible basis for arrhythmia. Currently our understanding of the electrophysiological basis of this less common but potentially lethal arrhythmia is limited. We are now evaluating different protocols involving early treatment with drugs, cooling of the patient, and atrioventricular sequential pacing.

Arrhythmias during intermediate follow up seemed to be related to haemodynamic condition. Interestingly, they occurred most often when treatment with diuretics was being tapered off either after the original Fontan operation or in two patients after a second operation. We could not determine whether reducing the dose more slowly would have avoided the postoperative arrhythmia. In nine of 11 patients sinus rhythm could be restored with medication. Multivariate analysis identified older age, increased right atrial size, and raised preoperative pulmonary artery pressure as risk factors for tachycardia during medium term follow up. It will be important to determine whether the incidence of arrhythmias increases with longer follow up, as in atrial repair of transposition of the great arteries, ${ }^{23}$ and how it relates to preoperative factors such as palliation and timing of the Fontan operation. Only $32 \%(25 / 78)$ of our patients were followed for five years or more after operation. Our multivariate analysis indicated the incidence of supraventricular extrasystoles on ambulatory monitoring increased with time since repair. This accords with evidence from a large group treated at the Mayo Clinic which suggests that late arrhythmias may be an increasingly important complication during follow up after the Fontan operation. ${ }^{11}$ In a retrospective study it is difficult to separate the effects of length of follow up from era of surgery and changes in management policy. We are currently prospectively studying preoperative, perioperative, and serial follow up ambulatory electrocardiographic monitoring of patients after Fontan repair.

Late tachycardias were well tolerated by most of our patients and none of the late deaths was provoked by an arrhythmia. Only one patient reported syncope in association with impaired systemic ventricular function. However, despite lack of immediate symptoms, loss of stable sinus rhythm may have adverse long term effects. We were able to study ventricular size and function in a group of patients before and during follow up after Fontan. ${ }^{24}$ One patient who developed asymptomatic chronic atrial flutter during follow up showed a significant increase in ventricular dimensions and a decrease in contractility after the onset of arrhythmia. This contrasted with all the other patients studied in sinus rhythm who showed a decrease in dimensions and increase in contractility studied by load-independent indices of ventricular function after operation. Thus preservation and restoration of sinus rhythm or at least maintaining an acceptable ventricular rate is important for long term postoperative wellbeing.

Ambulatory electrocardiographic monitoring showed an increased incidence of atrial and ventricular extrasystoles compared with nor- mal patients of similar age. ${ }^{10}$ The clinical significance of this finding could not be evaluated in our study because we did not perform serial monitoring. As in previous studies of congenital heart disease, very few arrhythmias were recorded by ambulatory monitoring that had not been manifested by clinical follow up or standard serial electrocardiography. Likewise, submaximal exercise testing, while yielding interesting data on the determinants of cardiac output after Fontan, ${ }^{25}$ provoked almost no arrhythmia. In none of our patients was the response of cardiac output to submaximal exercise testing over a physiological range limited by the inability to increase heart rate to appropriate values achieved by age matched controls. Currently, therefore, there does not seem to be an indication for prophylactic antiarrhythmic treatment based on findings of either ambulatory monitoring or exercise testing, especially bearing in mind the negative inotropic effects of most of such agents.

Based on our understanding of the Fontan circulation we may need to refine the indications for Fontan procedures, to alter the surgical technique on individual patients, or to consider other therapeutic strategies. The incidence of early and late arrhythmia has been an impetus to modify our surgical approach to the Fontan operation. In vitro and in vivo studies suggest several practical and theoretical advantages for the total cavopulmonary connection over the atriopulmonary connection in some patients. ${ }^{26}$ In the total cavopulmonary connection blood from the cavae is channelled to the pulmonary artery, either by a graft or a composite channel made from prosthetic material and sinus venosus wall. Therefore a smaller atrial mass is exposed to a chronic increase in systemic venous pressure and the stress in the atrial wall is lower (law of Laplace). The coronary sinus is left in the low pressure chamber and surgery occurs well away from the atrioventricular node. We are currently conducting a prospective study using perioperative and follow up ambulatory monitoring to examine whether this modification will reduce the incidence of early and late arrhythmias after the Fontan operation.

\section{Conclusions}

In a consecutive series of patients undergoing a Fontan operation, perioperative tachycardias were common and associated with significant morbidity and mortality. Early atrial flutter was a marker of adverse preoperative and postoperative haemodynamic function whereas $\mathrm{His}$ bundle tachycardia was not. During intermediate follow up development of supraventricular arrhythmias was also associated with borderline haemodynamic condition and with increased right atrial dimensions. Late tachycardias were haemodynamically better tolerated than in the early postoperative period. Modifications of the surgical technique designed to diminish energy loss in the circulation and prevent right atrial distension and increased pressure may reduce the incidence of early and late arrhythmias. 
MG was supported by the University of Leuven, Belgium

1 Fontan F, Baudet E. Surgical repair of tricuspid atresia. Thorax 1971;26:240-8.

2 Kirklin J, Barratt-Boyes B. Cardiac Surgery. New York: John Wiley \& Sons, 1986:866-72.

3 Bull C. Atrial and ventricular dependent circulations. In: Macartney FJ, ed. Congenital heart disease. Lancaster: MTP Press, 1986:35-53.

4 Ullal R, Anderson RH, Lincoln C. Mustard's operation modified to avoid dysrhythmias and pulmonary and systemic venous obstruction. J Thorac Cardiovasc Surg 1979; 78:431-9.

5 Gilette P, Kugler J, Garson A, Gutgesell H, Duff D, McNamara D. Mechanisms of cardiac arrhythmias after the Mustard operation for transposition of the great arteries. Am J Cardiol 1980;45:1225-30.

6 Boyden PA, Hoffman B. The effects of atrial electrophysiology and structure of surgically induced right atrial physiology and structure of surgically induced righ
enlargement in dogs. Circ Res 1981;49:1319-31.

7 Boyden R, Tilley L, Rham T, Liu S, Fenoglio J, Wit A Effects of left atrial enlargement on atrial transmembran potentials and structure in dogs with mitral valve fibrosis. Am J Cardiol 1982;49:1896-909.

8 Porter C, Battiste C, Humes R, et al. Risk factors for supraventricular tachyarrhythmias after Fontan procedure for tricuspid atresia [abstract]. Am Heart J 1986; 112:645.

9 Choussat A, Fontan F, Besse P, Vallot F, Chauve A, Bricaud $H$. Selection criteria for Fontan's procedure. In: Anderson RH, Shinebourne EA, eds. Pediatric cardiology. Edinburgh: Churchill Livingstone, 1978:559-66.

10 Nagashima M, Matsushima M, Ogawa A, et al. Cardiac arrhythmias in healthy children revealed by 24 hour arrhythmias in healthy children revealed by 24 hour ambulat $103-8$.

11 Humes R, Porter C, Mair D, et al. Intermediate follow-up and predicted survival after the modified Fontan procedure for tricuspid atresia and double-inlet ventricle. Circulation 1987;76(suppl III):67-71.

12 Dickinson D, Wilkinson J, Anderson K, Smith A, Ho S Anderson RH. The cardiac conduction system in situ ambiguus. Circulation 1979;59:879-85.

13 Ten Eick R, Singer D. Electrophysiological properties of diseased human atrium. I. Low diastolic potential and altered cellular response to potassium. Circ Res 1979; 44:545-57.

14 Puga F, Chiavarelli M, Hagler D. Modifications of the
Fontan operation applicable to patients with left atrioventricular valve atresia or single atrioventricular valve. Circulation 1987;76(suppl III):53-60.

15 Matculation 1987 , Kawashima Y, Kishimoto $H$, et al. Problems in the modified Fontan operation for univentricular heart of the right ventricular type. Circulation 1987;76(suppl III):45-52.

16 Alboliras E, Porter C, Danielson G, et al. Results of the modified Fontan operation for congenital heart lesions in patients without preoperative sinus rhythm. J Am Coll Cardiol 1985;6:228-33.

17 Barber G, Di Sessa T, Child J, et al. Hemodynamic responses to isolated increments in heart rate by atrial pacing after a Fontan procedure. Am Heart J 1988; 115:837-41.

18 Juaneda E, Haworth SG. Pulmonary vascular structure in patients dying after a Fontan procedure: the lung as a risk factor. Br Heart J 1984;52:575-80.

19 Haworth S. Pulmonary vasculature In: Anderson RH, Macartney FJ, Shinebourne EA, Tynan M, eds. Macartney FJ, Shinebourne EA, Tynan M, eds. Paediatric cardi

20 Rabinovitch M, Sanders S, Castaneda A, Reid L. Morphometric analysis of lung biopsy tissue in candidates for Fontan-type surgical procedures [abstract]. $\mathrm{Am} \mathrm{J} \mathrm{Cardiol}$ $1981 ; 47: 447$.

21 Harris P, Segal N, Bishop J. The relation between pressure and flow in the pulmonary circulation in normal subjects and in patients with chronic bronchitis and mitral stenosis. Circulation Res 1968;2:73-83.

22 Wolff G, Talmer D. Arrhythmias following cardiac surgery. In: Roberts NK, Gelband H, eds. Cardiac arrhythmia in the neonate, infant and child. Norwalk: Appleton Century Crofts, 1983:268-9.

23 Flin C, Wolff G, Macdonald D II, et al. Cardiac rhythm after the Mustard operation for complete transposition of the the Mustard operation for complete transposition

24 Gewillig M, Lundstrom U, Bull C, et al. Impact of the Fontan operation on left ventricular size and contractility Fontan operation on left ventricular size and con

25 Gewillig M, Lundström U, Wuse R, Bull C, Deanfield J. Exercise responses in patients after Fontan repair: patterns and determinants of performance. J Am Coll Cardiol 1990;15:1424-32.

26 De Leval M, Kilner P, Gewillig M, Bull C. Total cavopulmonary connection: a logical alternative to atriopulmonary connection for complex Fontan operations. J Thorac Cardiovasc Surg 1988;96:682-95. 